\title{
Subcellular anti-atherosclerotic therapy
}

\author{
Anastasia V. Ryabova1, Igor D. Romanishkin ${ }^{1}$, Aleksey S. Skobeltsin', Arkadii S. Moskalev¹, Vladimir I. \\ Makarov1, Victor B. Loschenov', Nikita G. Nikiforov², Igor A. Sobenin², Alexander N. Orekhov² \\ 1Prokhorov Institute of General Physics, Russian Academy of Sciences, Moscow 125315, Russia. \\ ${ }^{2}$ Institute of General Pathology and Pathophysiology, Moscow 125315, Russia.
}

Correspondence to: Dr. Alexander N. Orekhov, Laboratory of Angiopathology, Institute of General Pathology and Pathophysiology, Moscow 125315, Russia. E-mail: a.h.opexob@gmail.com

How to cite this article: Ryabova AV, Romanishkin ID, Skobeltsin AS, Moskalev AS, Makarov VI, Loschenov VB, Nikiforov NG, Sobenin IA, Orekhov AN. Subcellular anti-atherosclerotic therapy. Vessel P/us 2019;3:17.

http://dx.doi.org/10.20517/2574-1209.2019.10

Received: 12 Feb 2019 First Decision: 17 Feb 2019 Revised: 17 Feb 2019 Accepted: 17 Feb 2019 Published: 22 May 2019

Science Editor: Alexander N. Orekhov Copy Editor: Cai-Hong Wang Production Editor: Huan-Liang Wu

\section{Dear Editor,}

Photo-theranostics is a therapeutic and diagnostic approach that uses photosensibilization ${ }^{[1]}$. This approach makes part of the medicine of the future-subcellular therapy, including at the level of mitochondria ${ }^{[2]}$. Phototheranostics targets cells and tissues that are affected by pathological changes inducing apoptotic cell death. However, the approach allows targeting cellular organelles, if the photo-sensitizing agent is accumulated selectively. Such selectivity can result from the organelle's functional state, as in the case of mitochondria affected by mitochondrial genome abnormalities. Currently, subcellular therapy is being developed mainly for cancer cells, but we believe that such approaches are applicable to cardiology, in particular, for atherosclerotic diseases. For instance, some pro-atherogenic mitochondrial DNA mutations associated with atherosclerosis can lead to mitochondrial dysfunction ${ }^{[3]}$.

Some photo-sensitizing agents accumulate selectively in mitochondria making the photodynamic targeting efficient enough. Thus, protoporfyrine IX (PPIX), which is widely used in clinical practice, is produced from a prodrug 5-aminolevulinic acid (5-ALA) in course of heme synthesis cascade in the mitochondria. Exogenous 5-ALA causes excessive formation of PpIX, which cannot be efficiently processed by ferrochelatase and accumulates in the cells. PpIX accumulation directly depends on the cell's metabolic activity. 


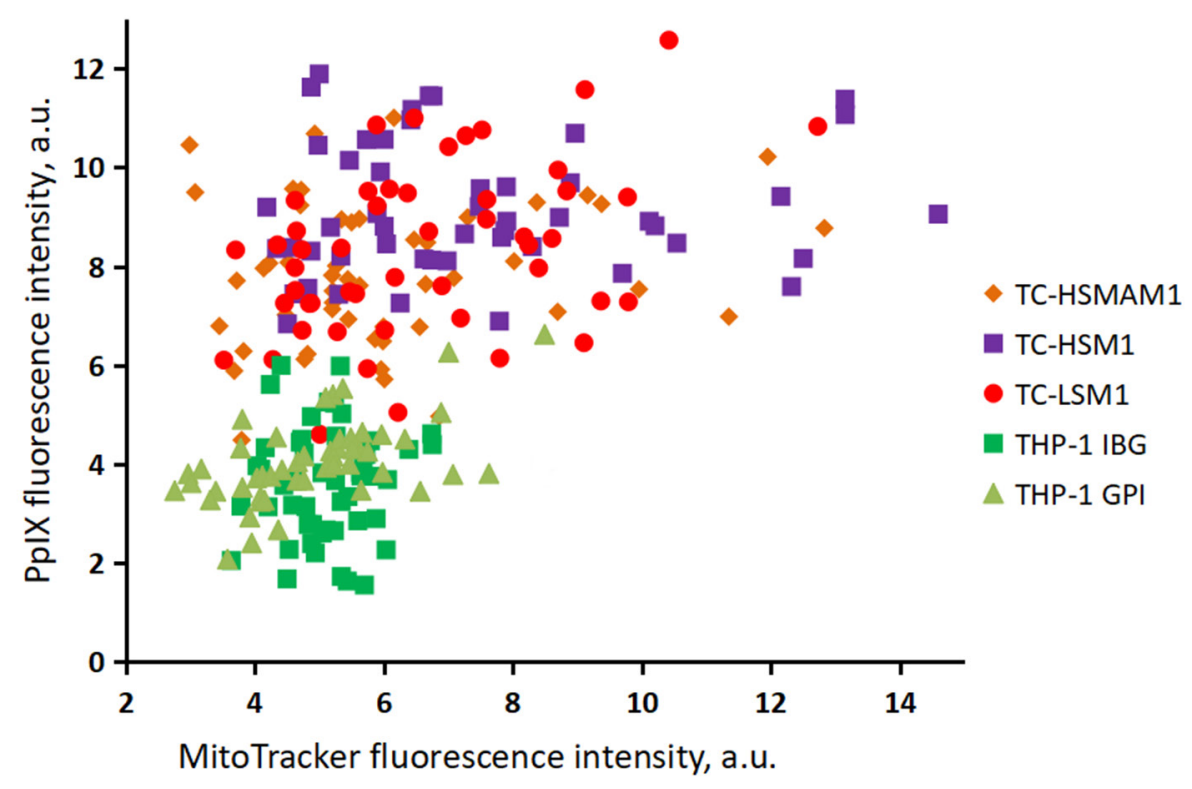

Figure 1. Distribution of protoporfyrine IX (PpIX) and MitoTracker fluorescence intensity in cybrid lines and THP-1 macrophage lines after $4 \mathrm{~h}$ of incubation with 5 -aminolevulinic acid

We studied 5-ALA-induced PpIX accumulation on cybrid lines derived from rhoo THP-1 macrophages and patients' thrombocytes with mutation-bearing proatherosclerotic mitochondria. We used 3 cybrid lines (TCHSMAM1, TC-HSM1, TC-LSM1) and 2 native THP-1 monocyte cultures obtained from different sources (THP-1 IBG, THP-1 GPI). The mitochondrial functional state was assessed as mitochondrial membrane potential using fluorescence intensity of mitochondrial dye MitoTracker ${ }^{\mathrm{TM}}$ Orange CMTMRos (Thermo Fisher Scientific). A high level of fluorescence is characteristic of active mitochondria; on the contrary, dysfunctional mitochondria possess a low level of fluorescence. Estimation of fluorescence was performed by laser scanning microscopy with spectral unmixing. MitoTracker and PpIX dyes were excited with a $561 \mathrm{~nm}$ laser, and fluorescence was detected at 570-750 nm wavelength. The signal was spectrally unmixed to obtain separate MitoTracker and PpIX signals. Individual cells areas were traced on microscopic images taken under identical conditions, and mean values of PpIX and MitoTracker fluorescence intensity were obtained for each cell.

We found that PpIX accumulation varied significantly from cell to cell within cell populations. Cybrid lines had increased PpIX accumulation in comparison with native THP-1 macrophage lines. A positive correlation between PpIX and mitochondrial potential was observed [Figure 1].

It can be assumed that selective elimination of dysfunctional (mutated) mitochondria can be achieved by adjusting laser intensity sufficient to induce photodynamic destruction of the organelles, while active (normal) mitochondria and the cells as a whole should be preserved at the used intensities.

Photodynamic therapy is a non-invasive treatment method in oncology, dermatology and infectious diseases. The disadvantage of this approach is the low depth of penetration into the tissue of excitation light and insufficient accumulation of photosensitizers in subcellular organelles. On the other hand, subcellular organelles, and in particular mitochondria, are promising therapeutic targets for effective therapy. At the same time, targeting mitochondria approaches do not necessarily have to be associated with the use of laser. It can be assumed that substances accumulating in mitochondria as a result of metabolic disturbances themselves without a laser will contribute to the death of dysfunctional mitochondria if there is an intramitochondrial overload of such substances. In addition to 5-ALA, there are many other mitochondria 
targeting substances. This is a good arsenal for a broad search for potential drugs for subcellular therapy including anti-atherosclerotic therapy ${ }^{[4,5]}$.

\section{DECLARATIONS}

\section{Authors' contributions}

Conducting experiments: Ryabova AV, Romanishkin ID, Skobeltsin AS, Moskalev AS, Makarov VI, Nikiforov NG

Manuscript preparation: Loschenov VB, Orekhov AN

Manuscript editing: Sobenin IA

\section{Availability of data and materials}

Not applicable.

\section{Financial support and sponsorship}

This work was supported by the Russian Science Foundation (Grant \# 19-15-00010).

\section{Conflicts of interest}

All authors declared that there are no conflicts of interest.

\section{Ethical approval and consent to participate}

Not applicable.

\section{Consent for publication}

Not applicable.

\section{Copyright}

(c) The Author(s) 2019.

\section{REFERENCES}

1. Cai Y, Si W, Huang W, Chen P, Shao J, et al. Organic dye based nanoparticles for cancer phototheranostics. Small 2018;14:e1704247.

2. Kang HC. Mitochondria-targeting theranostics. Biomater Res 2018;22:34.

3. Sazonova MA, Ryzhkova AI, Sinyov VV, Galitsyna EV, Orekhova VA, et al. New markers of atherosclerosis: a threshold level of heteroplasmy in mtDNA mutations. Vessel Plus 2017;1:182-91.

4. Orekhov AN, Ivanova EA. Introduction of the special issue "atherosclerosis and related diseases". Vessel Plus 2017;1:163-5.

5. Nikiforov NG, Galstyan KO, Nedosugova LV, Elizova NV, Kolmychkova KI, et al. Proinflammatory monocyte polarization in type 2 diabetes mellitus and coronary heart disease. Vessel Plus 2017;1:192-5. 